\title{
3D Guided Implant Surgery: A Case Report
}

\author{
Maharjan $\mathrm{SK}^{1 *}$, Mathema SRB ${ }^{2}$, Shrestha $\mathrm{SM}^{3}$ \\ ${ }^{1}$ Assistant Professor, ${ }^{2}$ Professor, Department of Prosthodontics and Maxillofacial Prosthetics \\ ${ }^{3}$ Professor, Department of Periodontics and Oral Implantology \\ People's Dental College and Hospital, Shorakhutte, Kathmandu, Nepal.
}

\begin{abstract}
Dental implant has been established as one of the most predictable and conservative approaches for the rehabilitation of missing teeth. With the evolution of newer material and advancement in digital technology, specially in computed tomography, implant planning software and guided implant surgery, the outcome and success in implant dentistry has become more predictable. Computer aided design (CAD) technique is being integrated into treatment planning, and computer-assisted manufacturing (CAM) is becoming more popular for rehabilitation procedures. This case report illustrate the diagnosis, planning and application of 3D guided technology in dental implant surgery
\end{abstract}

Key words: Dental implant, 3D CBCT, Guided implant, Flapless surgery, Stereolithography

\section{Introduction}

$\mathrm{W}$ ith the rapid development in computer technology in the last few years, the developments of computer aided design/ computer assisted manufacturing (CAD/CAM) technologies have brought great revolution in clinical dentistry, more specifically in oral implantology ${ }^{1,2}$. One of the recent approaches with this revolution is 3D Guided Surgery. This treatment concept has been developed with the aid of three-dimensional (3D) computed tomography (CT) scan images and computer aided design/ computer aided manufacturing (CAD/CAM) technology. Special software allows clinicians to plan implants in the virtual 3D image that is constructed from CT data. This enables clinicians to make a treatment plan with anatomical and prosthetic consideration. The planned data of implant placement are

\section{*Corresponding Author}

Dr. Shyam K. Maharjan, Assistant Professor Department of Prosthodontics and Maxillofacial Prosthetics, People's Dental College and Hospital Shorakhuttee, Kathmandu, Nepal.

E-mail:maharjan_sk@hotmail.com then transferred to fabricate computer guided stereolithographic surgical template. This template makes it possible to place implants in a pre-planned position without raising a flap. ${ }^{3,4}$

The advantages of 3D Guided surgical protocol are its minimally invasive nature, accuracy of implant placement, predictability and less post surgical discomfort. It also reduced time required for definitive rehabilitation in compare with conventional implant placement procedures. The available bone can be evaluated and used optimally by avoiding bone graft, or at least planned beforehand to achieve the best clinical result. $^{5}$

This case report explain the use of computerassisted diagnostics by 3D CBCT, perform virtual implant planning, fabricate the stereolithographic surgical template and placement of the dental implant in pre planned site with the help of surgical guide.

\section{Case Report}

A 30 year-old female patient reported in Department of Prosthodontics and Maxillofacial Prosthetics, People's Dental College and 
Hospital, Kathmandu with a chief complaint of missing lower right back tooth. Intraoral examination revealed missing 46, with history of extraction four months back due to endodontic failure (Fig 1). After discussing various modalities for replacement of her missing tooth, patient had optioned for implantsupported crown with guided surgery

The case was then planned and executed according to guided surgery protocol as purposed by Straumann ${ }^{\circledR}$ Guided Surgery.

\section{Step 1 - Treatment plan}

Maxillary and mandibular diagnostic impressions were made with irreversible hydrocolloid impression material, poured in Type III dental stone and diagnostic mounting was done. Intraoral periapical radiograph (IOPA) and Orthopantomograph (OPG) were made to evaluate bony foundation over the implant site, proximity of vital structures and overall status of remaining dentition. Intraoral evaluation of hard and soft tissues was performed. Presence of adequate keratinized tissue and bone width of $4.5 \mathrm{~mm}$ support our plan for guided flapless surgery with stereolithographic surgical template for the implant placement.

\section{Step 2 - Scan prosthesis fabrication}

The cast was then scan using Sirona InLab InEos X5 laboratory scanner and virtual model was achieved. (Fig 2). The scan prosthesis was a radiopaque duplicate of the current clinical situation with the provisional teeth set-up. Prosthetic restoration was planned on this virtual model. Once satisfactory alignment and design of prosthesis was achieved, this soft copy file was saved for the treatment planning using a dedicated 3D CBCT software

\section{Step 3 - CBCT scanning}

CBCT of mandibular right quadrant was made with Orthophos XG 3D Dentsply Sirona machine to expose in 5"x5" section. CBCT was then evaluated with a Sirona Galileos implant software ver.1.9, dedicate software for the possibility of rehabilitation with an implant supported prosthesis. Soft copy images of virtual prosthesis planned cast and 3D images of CBCT images were then merged together with the help of certain common reference points for definite planning of fixture length, diameter and angulations. (Fig 3)

\section{Step 4 - Software-based planning and fabrication of the surgical template (open system approach)}

After the evaluation of edentulous site in CBCT with virtual prosthesis, Straumann bone level tapered Roxolid SLA implant of $4.8 \mathrm{~mm}$ in diameter and $10 \mathrm{~mm}$ length was planned (Fig 4).

For planning the surgical guide, sleeve system of Sirona - CEREC Guide drill keys was selected. In this case drill key L was selected with position D2 in $19 \mathrm{~mm}$. After conformation of implant and surgical guide, the soft copy of case plan was sent to the Proficient Dental Lab Pvt. Ltd. for fabrication of the surgical template. Formlabs Form 2 Stereolithographic 3D Printer was used to print the surgical guide in resin. (Fig 5)

\section{Step 5 - Surgery with Straumann ${ }^{\circledR}$ guided instruments \& guided implant insertion}

After assuring the fit of the surgical template intraorally, the implant surgery was performed as per protocol with the guided instruments sets in the Straumann ${ }^{\circledR}$ Guided Surgery Cassette. (Fig 6) The surgical protocol, provided along with the surgical template recommended the sequence of instruments required to prepare each implant site.

Under Local Anesthesia, the surgery was initiated with the mucosa punch (diameter 4.7 $\mathrm{mm}$ ) with $15 \mathrm{rpm}$ through the sleeves with surgical template. (Fig 7). Mucosa punch 
allowed blade free incision with minimum trauma. Next step was the use of milling cutter to achieve sufficient flat bone surface for the purpose of easy drilling in the following stage. Implant bed was then pre drilled with the Pilot Drill (diameter $2.2 \mathrm{~mm}$ ). Basic implant bed preparation was continued using the diameter $2.8 \mathrm{~mm}, 3.5 \mathrm{~mm}$ and finally with $4.2 \mathrm{~mm}$ Twist Drill PRO using the corresponding drill handles. The correct cylinder of the drill handle and the respective drill length as indicated in the surgical protocol recommended by the software was taken into consideration. This surgical guide assures correct osteotomy site preparation as pre-planned earlier

After the completion of flapless implant bed preparation (Fig 9) Straumann bone level tapered

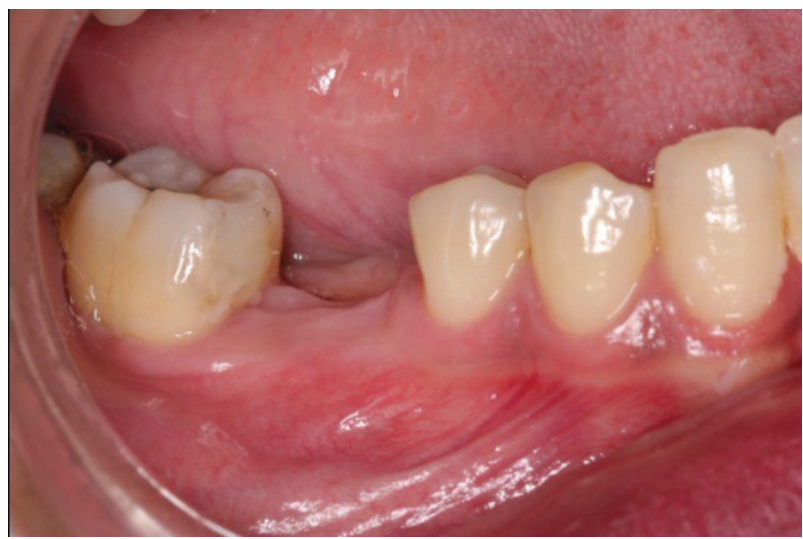

Figure 1: Intraoral condition with missing right mandibular first molar

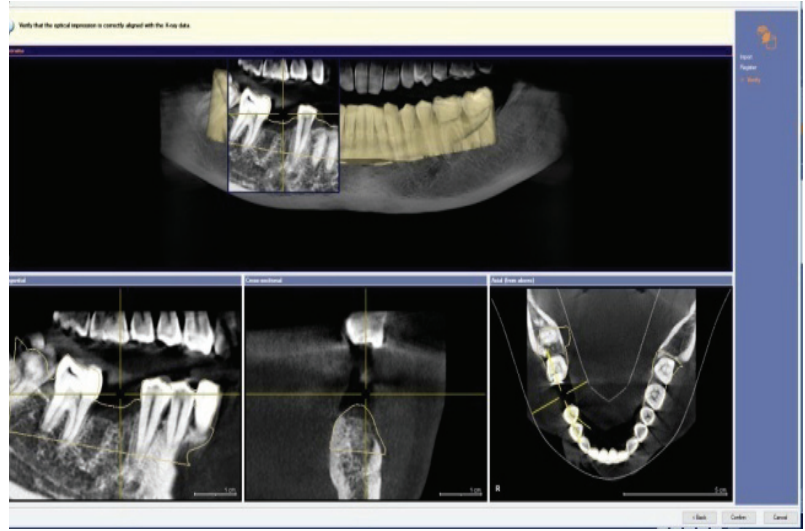

Figure 3: Merging of Virtual cast and CBCT images
Roxolid SLA implant (4.8 mm diameter and 10 $\mathrm{mm}$ length) was placed with 15 revolution per minute using surgical wrench. (Fig 10). Closure cap was then placed, which eliminate the need of suturing the overlying soft tissues on implant site. (Fig 11) Post surgical radiograph was made to ascertain the implant placement as per plan. The patient was recalled next day for follow up. It was observed that there was no pain, swelling or post surgical problems.

\section{Step 6 - Prosthetic procedures}

The definite prosthetic procedure for this case will be performed later after clinical and radiographic evidence of osseointregation.. Cement retained crown with the Solid Abutment System was planned for this case.

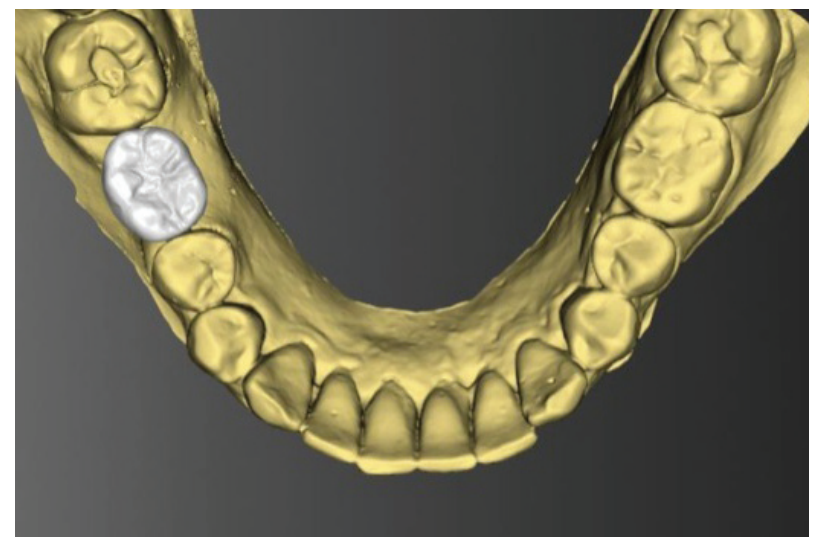

Figure 2: Fabrication of Scan Prosthesis in virtual cast

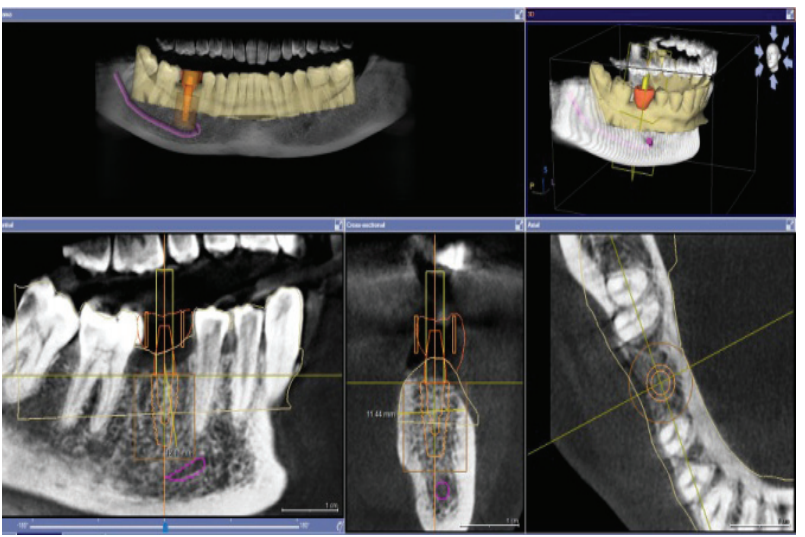

Figure 4: Planning of Dental Implant and Surgical Template using Sirona Galileos Implant software 


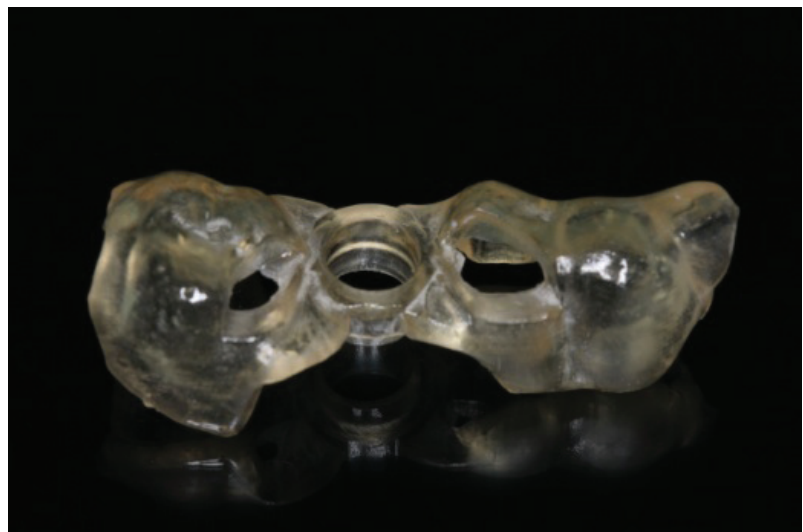

Figure 5: Printing of surgical template by using Formlabs Form 2 Stereolithographic 3D Printer

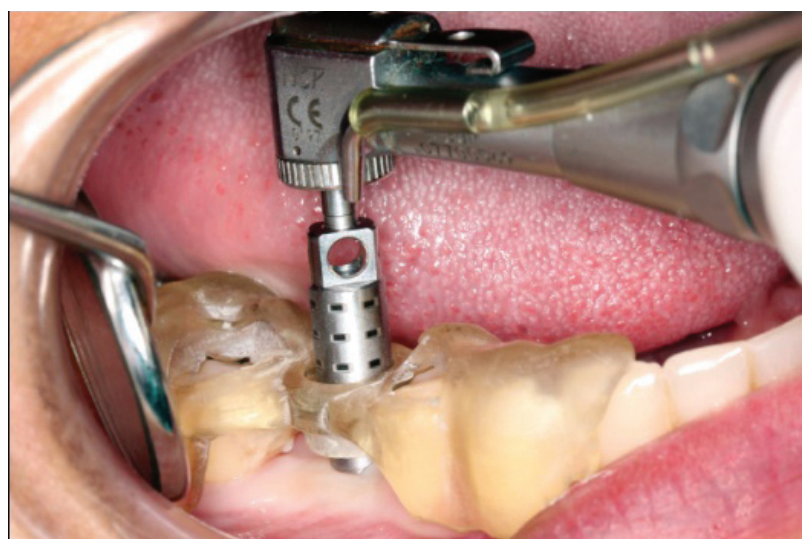

Figure 7: Mucosa Punch through surgical template

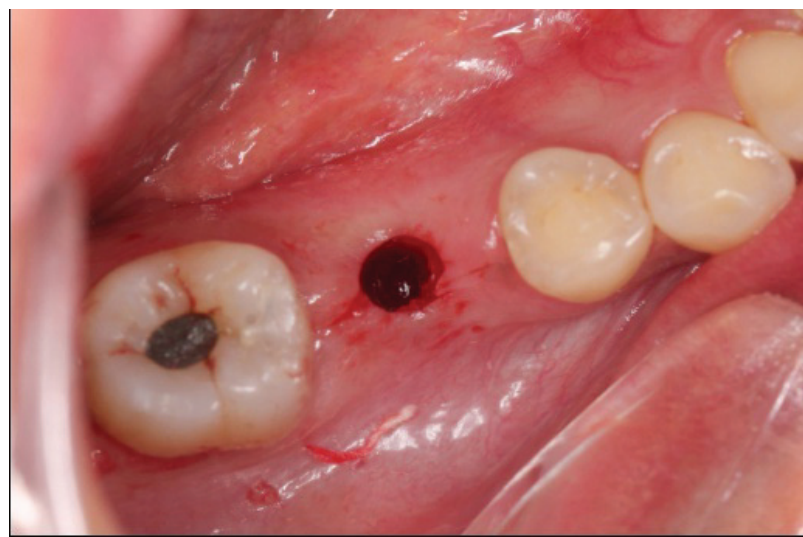

Figure 9: Minimal invasive Flapless implant site preparation

\section{Discussion}

Guided implant surgery using 3D CBCT and Stereolithographic surgical template is a relatively recent concept intended to facilitate the placement of dental implants by planning

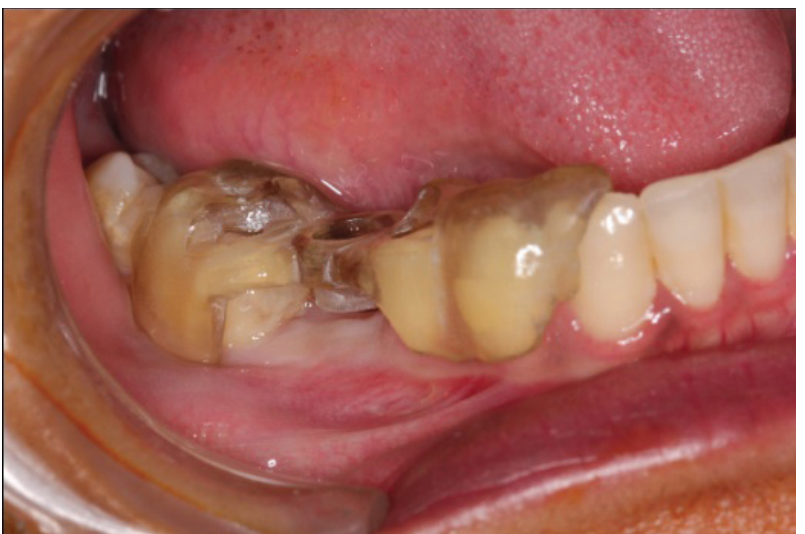

Figure 6: Checking the fit of Surgical template in patient mouth

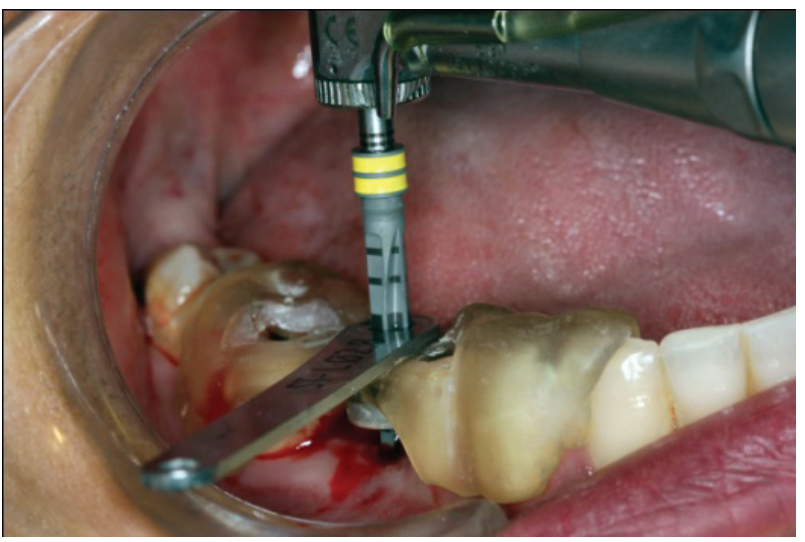

Figure 8: Implant Bed preperation with Twist Drill PRO for Guided Surgery.

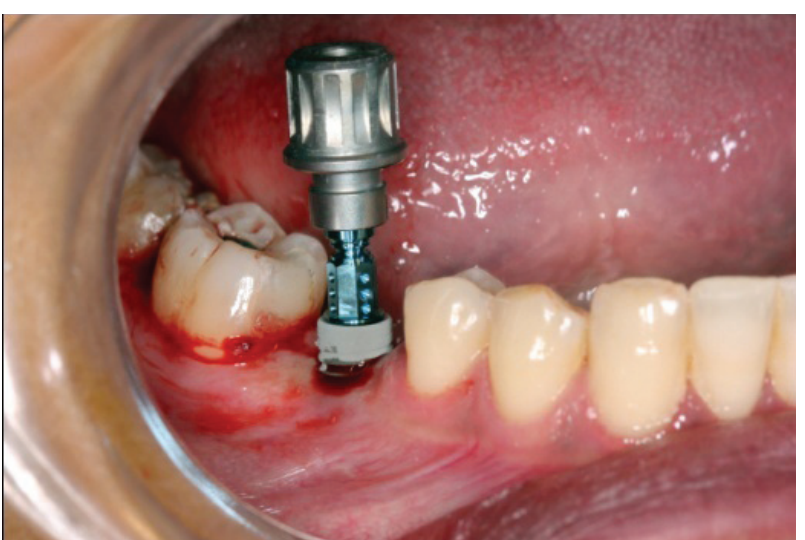

Figure 10: Straumann bone level tapered Roxolid SLA implant placement of $4.8 \mathrm{~mm}$ in diameter and $10 \mathrm{~mm}$

the final position of the implants prior to the surgery and the prosthetic work. However, as this is a fairly new concept, it is important to understand more about the technique and the final positioning of implants placed by the aid 


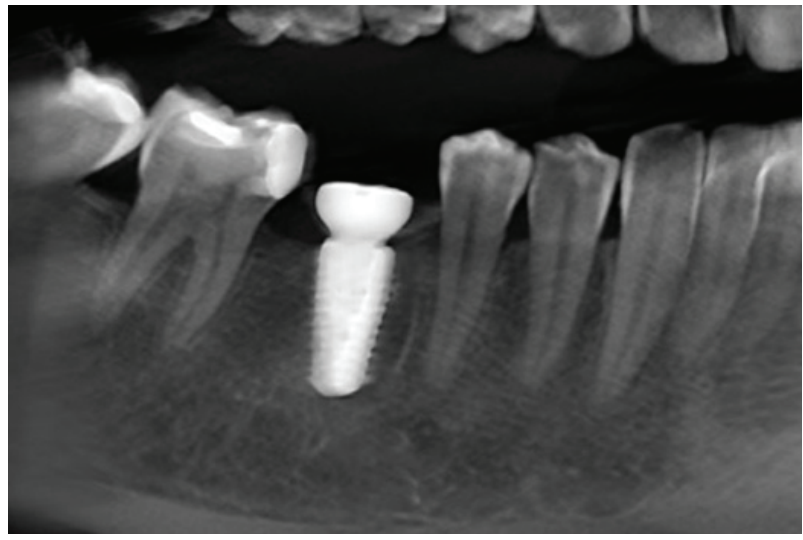

Figure 11: Radiographic evaluation after the placement of dental implant by 3D Guided Surgical Template

of a surgical template. ${ }^{6}$ Even if the duration of the surgical intervention may be shorter with flapless guided surgery compared to conventional techniques, it seems that much more time has to be invested in the preoperative planning. ${ }^{4}$

Clinicians need to learn about possible variations that could occur when placing implants with a CAD/CAM surgical template, to avoid anatomical risks, as well as for the final prosthetic reconstruction. When comparing clinical results from implants placed with CAD/CAM surgical templates with conventional approach, many studies showed similar or better results. Higher accuracy of surgical template is achieved from virtually designed and printed template after superimposing a surface scan with CBCT in order to transform the virtual plan into reality. If intraoral scans are additionally used instead of surface scans of a cast model after impression making, the accuracy may even improve as the intraoral scan may reduce the sources of inaccuracies associated with cast model preparation. ${ }^{5}$ Sebastian et al in 2015 stated that a high accuracy can be achieved using printed templates for guided implant surgery ${ }^{7}$

Several systems allow computer- guided implant placement with and without an open flap, or with and without immediate loading

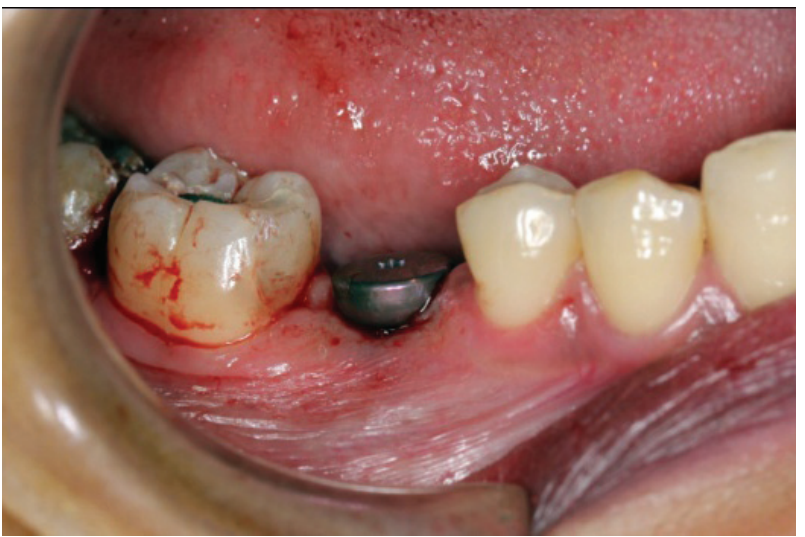

Figure 12: placement of gingival formor

are described in the literature but no obvious differences between conventional and guided implant treatments regarding implant survival rate. ${ }^{4}$ Susanne et al in 2011showedm precise transfer of implant replica position by means of simulated guided implant placement into a preoperative cast. ${ }^{8}$

The use of a computer program for planning prosthetically driven implant is highly efficient and safe. The three-dimensional view in this dedicated program allows the determination of the best implant position, the optimization of the implant axis, and the definition of the best surgical and prosthetic solution for the patient. Thus, a protocol that combines a computerguided technique with conventional surgical procedures becomes a promising option. ${ }^{9}$

The flapless guided implant placement techniques allow the surgeon to place the implants with minimal surgical trauma to the bone and associated soft tissues. In the study by Barter et al in 2010, patients were treated with flapless guided surgery to avoid a secondary exposure of earlier grafted sites. The implant survival rate was $98 \%$ and all prostheses were in use after 4 years. ${ }^{4}$ The flapless approach provide certain advantages such as maintaining better blood supply to site, reduced likelihood of resorption, maintains the soft tissue architecture 
and hard tissue volume at the site, decreases the surgical time, and accelerates recuperation. ${ }^{10}$

\section{Conclusions}

In this case report, missing mandibular right first molar was rehabilitated with dental implant supported prosthesis. Dental implant placement was planned prosthetically with the dedicated implant planning software using 3D CBCT and virtual cast; implant was placed in the planned position by flapless procedure using the stereo lithographic surgical template. Placement of the implant in the pre planned position was successfully performed. Patient experience less pain and discomfort. The whole procedure was less time consuming than the conventional procedure. 3D guided implant surgery will be the one of the routine procedures for rehabilitation of missing dentition in the days to come.

\section{References}

1. G Dev, Ottria L, Bollero P, et al. Aesthetic and functionality in fixed prosthodontic: sperimental and clinical analysis of the CADCAM systematic 3Shape. Oral Implantol (Rome) 2008;1(3-4):104-15.

2. Marchack CB. An immediately loaded CAD/ CAM-guided definitive prosthesis: a clinical report. J Prosthet Dent 2005;93(1):8-12.

3. G Dev, Ferraris F, Arcuri L, Guzzo F, Spinelli D. A novel workflow for computer guided implant surgery matching digital dental casts and CBCT scan. Oral Implantol (Rome) 2016;9(1):33-48.
4. Hultin M, Svensson KG, Trulsson M. Clinical advantages of computer-guided implant placement: a systematic review. Clin Oral Implants Res 2012;23 Suppl 6:124-35.

5. Kernen F, Benic GI, Payer M, et al. Accuracy of Three-Dimensional Printed Templates for Guided Implant Placement Based on Matching a Surface Scan with CBCT. Clin Implant Dent Relat Res 2016;18(4):762-8.

6. Pettersson A, Komiyama A, Hultin M, Nasstrom K, Klinge B. Accuracy of virtually planned and template guided implant surgery on edentate patients. Clin Implant Dent Relat Res 2012;14(4):527-37.

7. Kuhl S, Payer M, Zitzmann NU, Lambrecht JT, Filippi A. Technical accuracy of printed surgical templates for guided implant surgery with the coDiagnostiX software. Clin Implant Dent Relat Res 2015;17 Suppl 1:e177-82.

8. Platzer S, Bertha G, HeschlA, Wegscheider WA, Lorenzoni M. Three-dimensional accuracy of guided implant placement: indirect assessment of clinical outcomes. Clin Implant Dent Relat Res 2013;15(5):724-34.

9. Katsoulis J, Pazera P, Mericske-Stern R. Prosthetically driven, computer-guided implant planning for the edentulous maxilla: a model study. Clin Implant Dent Relat Res 2009;11(3):238-45.

10. Robinson $\mathrm{K}$ Job SL, R Ravi Chandran, K Harsha Kumar. Flapless Surgery Followed by Immediate Loading of Implant: A Blissful Combination of New Era. IJSS Case Reports \& Reviews; 2016. p. 7-10. 